\title{
Oxidação da pirita e seus efeitos em argamassas de cimento Portland sujeitas ao ataque por sulfatos de origem interna
}

\section{Pyrite Oxidation and its effects on Portland cement mortars subject to internal sulfates attack (ISA)}

\author{
Eduardo Pereira ${ }^{1}$, Kleber Franke Portella ${ }^{2}$, \\ Mariana d'Orey Gaivão Portella Bragança ${ }^{2}$, \\ Lucas Maier Ochiliski ${ }^{2}$, Isabela Cristina Oliveira ${ }^{2}$
}

\author{
${ }^{1}$ Av. Carlos Cavalcanti, 4748, Uvaranas, Ponta Grossa, PR CEP: 84030-900 \\ e-mail: eduardopereira@uepg.br \\ ${ }^{2}$ Endereço dos outros autores: \\ e-mail: portella@lactec.org.br; mariana.portella@lactec.org.br; llucasmaier@gmail.com; isabela.oliveira@copel.com
}

\begin{abstract}
RESUMO
$\mathrm{O}$ ataque por sulfatos de origem interna é resultante da reação química entre os íons $\mathrm{Fe}^{2+}$ e $\mathrm{SO}_{4}{ }^{2-}$, provenientes da oxidação de agregados sulfetados, e os compostos da pasta cimentícia, e tende a promover a degradação do concreto devido formação de produtos expansivos que geram fissuração e desagregação do material. Neste trabalho, a morfologia da pirita, presente em alguns tipos de agregado, foi avaliada. O mineral foi utilizado em substituição parcial à areia (10\%, em massa) na dosagem de argamassas, para estudo das modificações microestruturais e da variação dimensional linear sob envelhecimento natural durante 42 dias. A caracterização microestrutural foi executada em equipamento FEG/SEM com sonda analítica de EDS. Verificouse, na superfície da pirita oxidada, a formação de óxidos de ferro, os quais afetaram a interface entre a pasta de cimento e o agregado nos materiais produzidos com a pirita. Quanto a variação dimensional, a argamassa apresentou apenas contrações durante o período de estudo. Verificou-se a ocorrência de fissuração e a predominância na formação de cristais aciculares de etringita aos 42 dias enquanto aos 90 dias a presença de gipsita predomina. Óxidos de ferro oriundos da oxidação da pirita são evidentes nas argamassas aos 90 dias.
\end{abstract}

Palavras-chave: Pirita, oxidação, sulfatos, ataque interno por sulfatos, argamassa.

\begin{abstract}
The internal sulfates attack (ISA) is resultant of the chemical reaction between the ions $\mathrm{Fe}^{2+}$ and $\mathrm{SO}_{4}{ }^{2-}$, by products of sulfide aggregates oxidation, and the composites of the cement paste. These actions promote the degradation of concrete due to the formation of expansive products that generates cracking and dissolution of the material. In this study, the morphology of the pyrite, present in some kinds of aggregate, was evaluated. In parallel, the mineral was utilized in partial replacement for sand (10\%, in mass) in mortar dosage, for study of microstructural modifications and linear dimensional variation under natural aging during 42 days. The microstructural characterization was executed in a FEG/SEM equipment with EDS analytical probe. On the surface of the oxidized pyrite were formed iron oxides, which affected the interface between the cement paste and the aggregate in the produced materials with the pyrite. As the dimensional variation, the mortar presented only contractions during the period of study. It was verified the occurrence of cracking and the predominance of ettringite acicular crystals formation at 42 days, while at 90 days the presence of gypsum predominates. Iron oxides from the oxidation of the pyrite were evident in mortars at 90 days.
\end{abstract}

Keywords: Pyrite, oxidation, sulfates, internal sulfate attack, mortar.

\section{INTRODUÇÃO}

As estruturas civis são caracterizadas por ter vida longa em serviço. Porém, para que suas propriedades estejam condizentes, há uma série de fatores que deve ser seguida, como por exemplo: i) as solicitações mecâni- 
cas, internas e externas ao concreto não podem ser superiores às suas resistências nominais (tração, compressão, flexão); ii) os seus insumos (aglomerantes e agregados) têm de ser compatíveis com o meio no qual os concretos e argamassas estarão inseridos, como para as regiões agressivas por íons sulfato, cloretos entre outros, utilizando-se cimentos especiais, traços com pozolana e com outras adições minerais; iii) a sua dosagem deve conter uma baixa relação água/aglomerante hidráulico; iv) os agregados graúdos e miúdos necessitam ter pequena ou nenhuma reatividade; e, v) a sua produção deve, naturalmente, ter uma baixa relação custo versus benefício. Em consequência, os problemas da engenharia são agravados quando da construção de grandes obras como as barragens de usinas hidroelétricas e de captação de água, uma vez que estas são realizadas em locais com aspectos geológicos diversos e, normalmente, fora dos grandes centros fornecedores de matérias-primas [1-4].

Por representar a maior quantidade em volume de uma estrutura de concreto, os agregados necessitam ter suas constituições física e química analisadas. Com o diagnóstico precoce da litologia e das suas possíveis contaminações mineralógicas e químicas, pode-se antever a produção de concretos com um mínimo de interferência na sua vida útil de projeto, uma vez que, devido a uma caracterização inadequada, muitas manifestações patológicas só são verificadas no decorrer do tempo de operação da grande obra [5-10], como são os casos das reações álcali agregado ou ataque interno por sulfatos, devido a contaminação do agregado por sulfetos minerais.

Agregados utilizados em obras de construção civil podem vir contaminados com outras fases químicas e mineralógicas, causadoras de reações deletérias ao concreto, como a pirita ou $\mathrm{FeS}_{2}$, já que este é um mineral comum na natureza. Por exemplo, na região sul do Brasil, este mineral está ligado aos folhelhos pirobetuminosos abundantes na Formação Irati da Bacia do Paraná, que compreende uma área equivalente a 1,5 milhões.m² explorados na América do Sul (Brasil, Argentina e Paraguai) [11].

Baseado no exposto, o objetivo deste artigo é avaliar o efeito da presença de pirita, um sulfeto mineral, no período de dormência de barras de argamassa de cimento Portland sujeitas ao ataque interno por sulfatos. Para isto, a morfologia da pirita oxidada foi discutida, frente aos produtos formados por estas reações e o comportamento de argamassas moldadas conforme a NBR 13583: 2014 [12] com substituição em 10\%, em massa, de uma areia de britagem, pelo referido agregado contendo a pirita $\left(\mathrm{FeS}_{2}\right)$ com foco na microestrutura do mineral e dos compostos formados na matriz cimentícia em decorrência da oxidação da pirita.

\section{REFERENCIAL TEÓRICO}

As reações de oxidação da pirita estão descritas por diversos autores [1, 7, 11, 13-18]. O mineral, quando

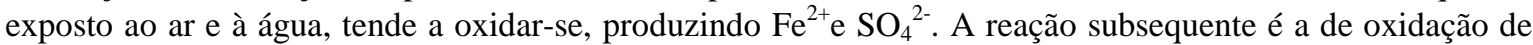
$\mathrm{Fe}^{2+}$ a $\mathrm{Fe}^{3+}$, que se hidrolisa para formar o hidróxido de ferro, uma fase pouco solúvel em pH maior que 3,5. Além disto, os sais de sulfato de ferro formados nesta etapa tendem a precipitar sobre as superfícies, alterando parcialmente as tonalidades da estrutura. A pirita pode ser oxidada também pelo íon férrico, que controla a taxa de oxidação da reação. Após o processo de oxidação da pirita pelo $\mathrm{O}_{2}$, que libera o íon ferroso, um ciclo de reação é estabelecido, no qual o íon férrico produzido é subsequentemente reduzido pela pirita, gerando $\mathrm{Fe}^{2+}$ e ácido sulfúrico $\left(\mathrm{H}_{2} \mathrm{SO}_{4}\right)$.

Estudos tratam a oxidação da pirita fazendo uma analogia com as reações envolvidas durante o processo de corrosão do ferro e da precipitação de seus óxidos [13, 15]. Na abordagem termodinâmica, a oxidação da pirita pode ser explicada por um mecanismo eletroquímico, no qual a reação anódica produz íons de ferro e de sulfato e a catódica reduz o oxidante (oxigênio ou os próprios íons ferro) [14].

Os principais fatores que interferem e aceleram o processo de transformação dos sulfetos são a umidade, o oxigênio, a temperatura, a superfície específica e a morfologia da pirita, o teor de ferro no mineral, a presença de bactérias aeróbias e o pH do sistema [19].

A degradação do concreto por íons sulfato ocorre a partir de sua reação química com os compostos hidratados do cimento os quais ficam, normalmente, disponibilizados nos poros [1, 6, 17-20]. Basicamente, três reações estão envolvidas: (i) a combinação de gipsita do cimento e dos aluminatos hidratados de cálcio com o monossulfato hidratado, formando a etringita $\left(\mathrm{CaO}_{3} \cdot \mathrm{Al}_{2} \mathrm{O}_{3} \cdot 3 \mathrm{CaSO}_{4} \cdot 32 \mathrm{H}_{2} \mathrm{O}\right)$; (ii) a combinação dos íons sulfatos com o hidróxido de cálcio livre e/ou o C-S-H, produzidos pela hidratação do cimento, formando gipsita $\left(\mathrm{CaSO}_{4}\right.$. $\left.2 \mathrm{H}_{2} \mathrm{O}\right)$; e, (iii) a combinação da gipsita e do carbonato de cálcio com o C-S-H (silicatos de cálcio hidratados), formando a taumasita $\left(\mathrm{CaCO}_{3} \cdot \mathrm{CaSiO}_{3} \cdot \mathrm{CaSO}_{4} \cdot 15 \mathrm{H}_{2} \mathrm{O}\right)$ [10, 21-22]. No concreto, a formação da gipsita está acompanhada da perda de adesão e da resistência mecânica, podendo haver ainda expansão e lascamento do material; a formação de etringita está associada a uma expansão e a uma fissuração da pasta e, adicionalmente, do agregado; e, a formação de taumasita, na transformação do concreto endurecido em uma massa não coesa, devido à dissolução do C-S-H, o principal composto da matriz cimentícia [9, 23].

Os produtos gerados durante a oxidação dos íons sulfeto e da posterior reação do íon sulfato com os 
produtos do cimento hidratado cristalizam-se, apresentando, na sua maioria, um volume maior do que os elementos que lhe deram origem, apesar dos estudos concentrarem-se na identificação dos compostos e inferirem sobre sua expansão. Este fenômeno provoca um aumento das pressões internas na estrutura, que pode levar, como comentado anteriormente, a uma expansão e, consequente, a uma fissuração do material [5-7; 24]. Estas reações podem comprometer a durabilidade do concreto, uma vez que este, ao fissurar perde suas características mecânicas e promove a formação de um caminho preferencial que possibilita a entrada de água e de mais contaminantes solúveis, os quais podem desencadear novas reações de deterioração ou ainda acelerar os processos que já estejam ocorrendo [25].

\section{MATERIAIS E MÉTODOS}

\subsection{Caracterização dos materiais}

O aglomerante utilizado para a dosagem das argamassas foi o cimento Portland CPII-F-32 (Cimento Portland com adição de fíler calcário). As características físico-químicas do cimento estão reunidas na Tabela 1, conforme dados fornecidos pelo fabricante.

Tabela 1: Caracterização físico-química do cimento CPII-F-32 [26]

\begin{tabular}{|c|c|c|c|c|c|c|c|c|c|c|}
\hline \multicolumn{11}{|c|}{ Análise Química por XRF, \% } \\
\hline $\mathrm{Al}_{2} \mathrm{O}_{3}$ & $\mathrm{SiO}_{2}$ & $\mathrm{Fe}_{2} \mathrm{O}_{3}$ & $\mathrm{CaO}$ & $\mathrm{MgO}$ & $\mathrm{SO}_{3}$ & $\begin{array}{l}\mathrm{CaO} \\
\text { Livre }\end{array}$ & $\begin{array}{l}\text { Equiva } \\
\text { Alcalin }\end{array}$ & $\begin{array}{l}\text { ente. } \\
\text { o }\end{array}$ & $\begin{array}{l}\text { Resíduo } \\
\text { Insolúvel }\end{array}$ & P. F. \\
\hline 4,43 & 18,83 & 2,64 & 60,68 & 4,98 & 2,83 & 0,73 & 0,6 & & 1,78 & 5,05 \\
\hline \multicolumn{11}{|c|}{ Análises físicas } \\
\hline \multicolumn{2}{|c|}{$\begin{array}{c}\text { Massa específica, } \\
\mathrm{g} / \mathrm{cm}^{3}\end{array}$} & \multicolumn{2}{|c|}{$\begin{array}{c}\text { Expansão } \\
\text { quente, mm }\end{array}$} & \multicolumn{2}{|c|}{$\begin{array}{l}\text { Início de pega, } \\
\text { h:min }\end{array}$} & \multicolumn{2}{|c|}{$\begin{array}{c}\text { Fim de pega, } \\
\text { h:min }\end{array}$} & \multicolumn{2}{|c|}{$\begin{array}{l}\text { Consistência } \\
\text { Normal, \% }\end{array}$} & $\begin{array}{l}\text { Blaine, } \\
\mathrm{cm}^{2} / \mathrm{g}\end{array}$ \\
\hline \multicolumn{2}{|c|}{3,11} & \multicolumn{2}{|c|}{0,50} & \multicolumn{2}{|c|}{$3: 35$} & \multicolumn{2}{|c|}{$4: 00$} & & 26,40 & 3,140 \\
\hline \multicolumn{6}{|c|}{ Distribuição granulométrica, \% } & \multicolumn{5}{|c|}{ Resistência mecânica à compressão, MPa } \\
\hline \multicolumn{3}{|c|}{ \# 200} & \multicolumn{3}{|l|}{ \# 325} & 1 dia & 3 dias & & 7 dias & 28 dias \\
\hline \multicolumn{3}{|c|}{3,00} & \multicolumn{3}{|c|}{14,70} & 13,7 & 26,9 & & 32,5 & 41,3 \\
\hline
\end{tabular}

Nota: P. F., perda ao fogo.

O agregado miúdo é uma areia de britagem de origem calcária. A massa específica do agregado miúdo foi determinada conforme a NBR NM 52: 2009 [27] e a absorção de água conforme NBR NM 30:2001 [28]. Os ensaios para a determinação da massa unitária e do volume de vazios dos agregados miúdos foram realizados segundo as diretrizes da NBR NM 45:2006 [29], enquanto que, o teor de material pulverulento foi determinado conforme proposto pela NBR NM 46: 2003 [30].

$\mathrm{O}$ agregado miúdo foi caracterizado por fluorescência de raios-X (XRF) e por difração de raios-X (XRD) para a verificação das suas fases químicas e mineralógicas presentes. Os ensaios de XRF foram realizados em espectrômetro PANalytical Axios Max e executados em pastilhas de pó prensados. A análise por XRD foi realizada em difratômetro PANalytical EMPYREAN, com pastilhas de pó prensados, com identificação mineralógica por comparação com os padrões do ICDD (International Centre for Diffraction Data). A medida foi realizada em varredura entre $5^{\circ}$ e $75^{\circ}$, em $2 \theta$ e tempo por passo de $1 \mathrm{~s}$, utilizando-se um tubo com ânodo de cobre, $40 \mathrm{kV} / 30 \mathrm{~mA}$ e fenda divergente de $1^{\circ}$. Os resultados de caracterização por XRD, indicam como constituintes principais do agregado miúdo, as fases químicas calcita $\left(\mathrm{CaCO}_{3}\right)$, quartzo $\left(\mathrm{SiO}_{2}\right)$ e dolomita $\left(\mathrm{CaMg}\left(\mathrm{CO}_{3}\right)_{2}\right)$. Na Tabela 2, estão apresentadas as propriedades do agregado miúdo, bem como sua caracterização por XRF.

Tabela 2: Caracterização física e química do agregado miúdo

\begin{tabular}{|c|c|c|c|c|c|c|}
\hline \multicolumn{2}{|c|}{$\begin{array}{c}\text { Massa específica } \\
\left(\mathrm{g} / \mathrm{cm}^{3}\right)\end{array}$} & $\begin{array}{l}\text { Absorção de } \\
\text { água (\%) }\end{array}$ & $\begin{array}{c}\text { Massa unitária } \\
\left(\mathrm{kg} / \mathrm{m}^{3}\right)\end{array}$ & $\begin{array}{l}\text { Índice de vazios } \\
\text { (\%) }\end{array}$ & \multicolumn{2}{|c|}{$\begin{array}{c}\text { Teor de material pulve- } \\
\text { rulento (\%) }\end{array}$} \\
\hline 2,66 & & 0,60 & 1720 & 35,3 & & \\
\hline \multicolumn{7}{|c|}{ Análise química do agregado miúdo, por XRF, \% em massa } \\
\hline $\mathrm{SiO}_{2}$ & $\mathrm{Fe}_{2} \mathrm{O}_{3}$ & $\mathrm{Al}_{2} \mathrm{O}_{3}$ & $\mathrm{CaO}$ & $\mathrm{TiO}_{2}$ & MgO & $\mathrm{Na}_{2} \mathrm{O}$ \\
\hline 5,84 & 0,97 & 1,69 & 45,47 & 0,10 & 4,29 & 0,03 \\
\hline $\mathrm{K}_{2} \mathrm{O}$ & $\mathrm{P}_{2} \mathrm{O}_{5}$ & $\mathrm{SO}_{3}$ & Mno & Sro & & \\
\hline 0,35 & 0,07 & --- & 0,04 & 0,17 & & \\
\hline
\end{tabular}


A pirita foi proveniente da região de Figueira - PR e consistiu em um resíduo de carvão mineral, com granulometria entre 9,5 e $19 \mathrm{~mm}$. Este material foi beneficiado em britador tipo mandíbula para a obtenção do passante na peneira de abertura de $2,4 \mathrm{~mm}$, obtendo-se granulometria similar à areia. Após, o material foi conservado em câmara seca $\left(\mathrm{T}=(23 \pm 2){ }^{\circ} \mathrm{C}\right.$ e de $\left.\mathrm{UR}=(50 \pm 5) \%\right)$ para minimizar os efeitos de sua oxidação superficial.

A mineral foi caracterizado por XRF e XRD utilizando-se procedimento similares ao adotado para o agregado miúdo. O resultado de sua caracterização química por XRF está apresentado na Tabela 3, na qual por cálculos estequiométricos identificou-se um teor médio de pirita em torno de $94 \%$, em massa. A fase química, pirita $\left(\mathrm{FeS}_{2}\right)$, foi identificada por XRD no mineral utilizado, com provável presença de traços de quartzo $\left(\mathrm{SiO}_{2}\right)$, gipsita $\left(\mathrm{CaSO}_{4} \cdot 2 \mathrm{H}_{2} \mathrm{O}\right)$ e calcita $\left(\mathrm{CaCO}_{3}\right)$.

Tabela 3: Caracterização química do agregado contendo a pirita, por XRF

\begin{tabular}{c|c|c|c|c|c|c|c|c|c|c|c}
\hline \multicolumn{1}{c}{ Análise química do mineral, por XRF, em \%, em massa } \\
\hline $\mathbf{S O}_{3}$ & $\mathbf{F e}_{2} \mathbf{O}_{3}$ & $\mathbf{S i O}_{2}$ & $\mathbf{C a O}$ & $\mathbf{A l}_{2} \mathbf{O}_{3}$ & $\mathbf{A s}_{2} \mathbf{O}_{3}$ & $\mathbf{Z n O}$ & $\mathbf{N a}_{2} \mathbf{O}$ & $\mathbf{K}_{2} \mathbf{O}$ & $\mathbf{T i O}_{2}$ & MnO & P.F \\
\hline 35,50 & 20,40 & 1,50 & 1,40 & 0,60 & 0,20 & 0,10 & 0,10 & $<0,1$ & $<0,1$ & $<0,1$ & 40,00 \\
\hline
\end{tabular}

Para a análise da oxidação da pirita, após o quarteamento de 1000 g do mineral, foram selecionados, aleatoriamente, fragmentos de agregados retirados da câmara seca. Os mesmos foram seccionados para a análise de sua morfologia por microscopia eletrônica de varredura com emissão de campo, antes (câmara seca) e após a sua exposição em ambiente laboratorial, buscando-se monitorar as mudanças superficiais decorrentes do processo oxidativo.

As imagens foram obtidas com o uso de um microscópio FEG SEM, Tescan, Mira 3, com detectores SE, BSE e microssonda analítica de raios-X Oxford X-Max 50 (EDS), com metalização das amostras.

\subsection{Moldagem e envelhecimento das barras de argamassa}

A metodologia de preparo dos corpos de prova de argamassa foi a recomendada pela norma NBR 13583: 2014 [12], com as seguintes exceções: i) a moldagem dos prismas de argamassa nas dimensões de ( 25 x 25x 285) $\mathrm{mm}$ foi realizada com um traço equivalente a 1 (uma) parte de cimento para 2,88 partes de areia e 0,32 de mineral contendo a pirita, ou seja, com 10\%, em massa, de areia, a qual foi substituída por este mineral.

Este percentual foi determinado com base nos trabalhos de Duchesne e Fournier [10], Gomides et al. [19], Lima [31], Hagerman [32], Wakizaka et al. [33], Faquineli [34], Bragança et al. [35], que apresentaram a existência de concentrações entre 1\% e 10\% de pirita em agregados utilizados em concreto e argamassa; e, ii) a avaliação do potencial de mitigação das expansões foi realizada pelo envelhecimento dos corpos de prova em ambiente atmosférico natural, por 42 dias, a uma temperatura entre $10{ }^{\circ} \mathrm{C}$ e de $30{ }^{\circ} \mathrm{C}$ e com uma umidade relativa entre 60 e 95\%, conforme dados do INMET [36], ao invés de suas imersões em solução aquosa de sulfato de sódio. Este ponto é relevante pois sendo as condições de exposição natural, as variantes climáticas influem na variabilidade dos resultados ao longo do tempo. A relação água/aglomerante foi de 0,60.

Para tanto, foram moldados 6 prismas de argamassa para o acompanhamento da evolução das reações químicas a partir da medida das expansões lineares e 4 prismas para a sua caracterização, em diferentes idades. As condições de preparo e de moldagem das amostras foram de $\mathrm{T}=(23 \pm 2){ }^{\circ} \mathrm{C}$ e de UR $>50 \%$. O traço base sugerido pela NBR 13583: 2014 [12] é de 1: 3,2: 0,60.

A cura dos corpos de prova foi feita em 2 etapas (NBR 13583: 2014) [12]: i) 2 dias em câmara úmida a uma condição de temperatura ambiente de $(22 \pm 2)^{\circ} \mathrm{C}$ e de UR >95\%; e de 12 dias em água saturada com cal, conforme recomendação normativa. Após finalizada a segunda etapa de cura, procederam-se às primeiras leituras do comprimento das barras, as quais foram estabelecidas como referência para as demais análises.

O pórtico comparador para a medida de variação de comprimento das barras de argamassa foi composto por um relógio comparador digital com resolução de $0,001 \mathrm{~mm}$, que foi calibrado com uma barra padrão de aço (Figura 1). As barras de argamassa ficaram expostas ao ambiente laboratorial $\left((22 \pm 2)^{\circ} \mathrm{C}\right.$ e de UR $>50 \%$ ), durante $1 \mathrm{~h}$, antes da execução das leituras, sendo os valores de expansão resultantes de 3 leituras por barra. 

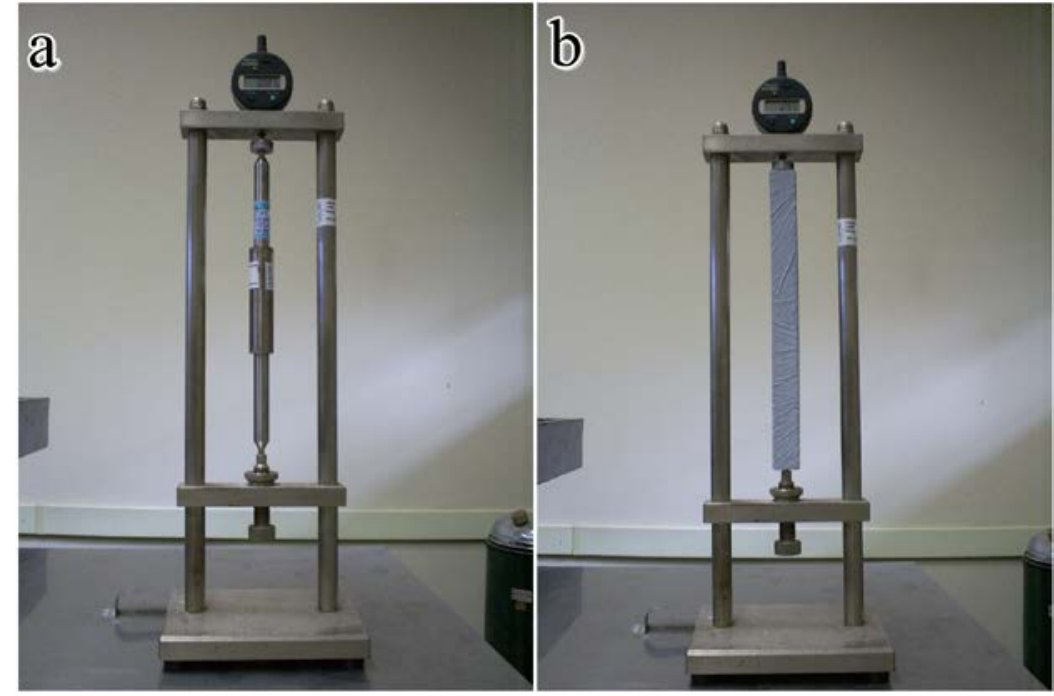

Figura 1: Pórtico com relógio comparador para leituras de variação linear em barras de argamassa: (a) com barra padrão de calibração e, (b) com barra de argamassa

Após 42 e 90 dias de envelhecimento natural, as amostras de argamassa foram fraturadas e analisadas na sua parte mais interna, utilizando-se os procedimentos de ensaio pelo FEG SEM, similares ao utilizado para a avaliação da oxidação da pirita.

\section{RESULTADOS E DISCUSSÃO}

\subsection{Oxidação da pirita}

Na Figura 2a, está apresentada a micrografia da pirita, na qual foi possível verificar a presença de cristais isométricos, hábito piritoédrico (dodecaedros com faces pentagonais) e a presença de carvão mineral entre os grãos da matriz. Como resultado médio de 12 espectros semiquantitativos de EDS, a composição do mineral à base de pirita natural (a partir da análise da superfície de fratura), foi de $(46,7) \%$ de $\mathrm{S}$, $(42,8) \%$ de Fe, $(5,8) \%$ de C e $(4,7) \%$ de demais compostos residuais (Al, O, Si, Na e K). Segundo Klein e Dutrow [4] e Gomides [16], a pirita pura é composta por $46,6 \%$ de ferro e $53,4 \%$ de enxofre, podendo conter impurezas. A presença de carbono e dos demais elementos é decorrente da origem da adição, a qual é um resíduo de carvão mineral (pirobetuminoso proveniente da Bacia do Paraná).

Em relação à média, a composição química básica da pirita analisada por EDS foi de 52,3\% em enxofre e de $47,9 \%$ de ferro, ou seja, com um erro percentual experimental máximo de $3 \%$ em relação ao valor teórico. Já, na Figura 2b, está apresentada a micrografia da amostra exposta por, aproximadamente, 90 dias de armazenamento em condições laboratoriais $\left(\mathrm{T}=(22 \pm 2){ }^{\circ} \mathrm{C}\right.$ e de UR $\left.>50 \%\right)$ onde se pode verificar a ocorrência de mudanças morfológicas no mineral, com um possível processo de oxidação superficial da pirita. 


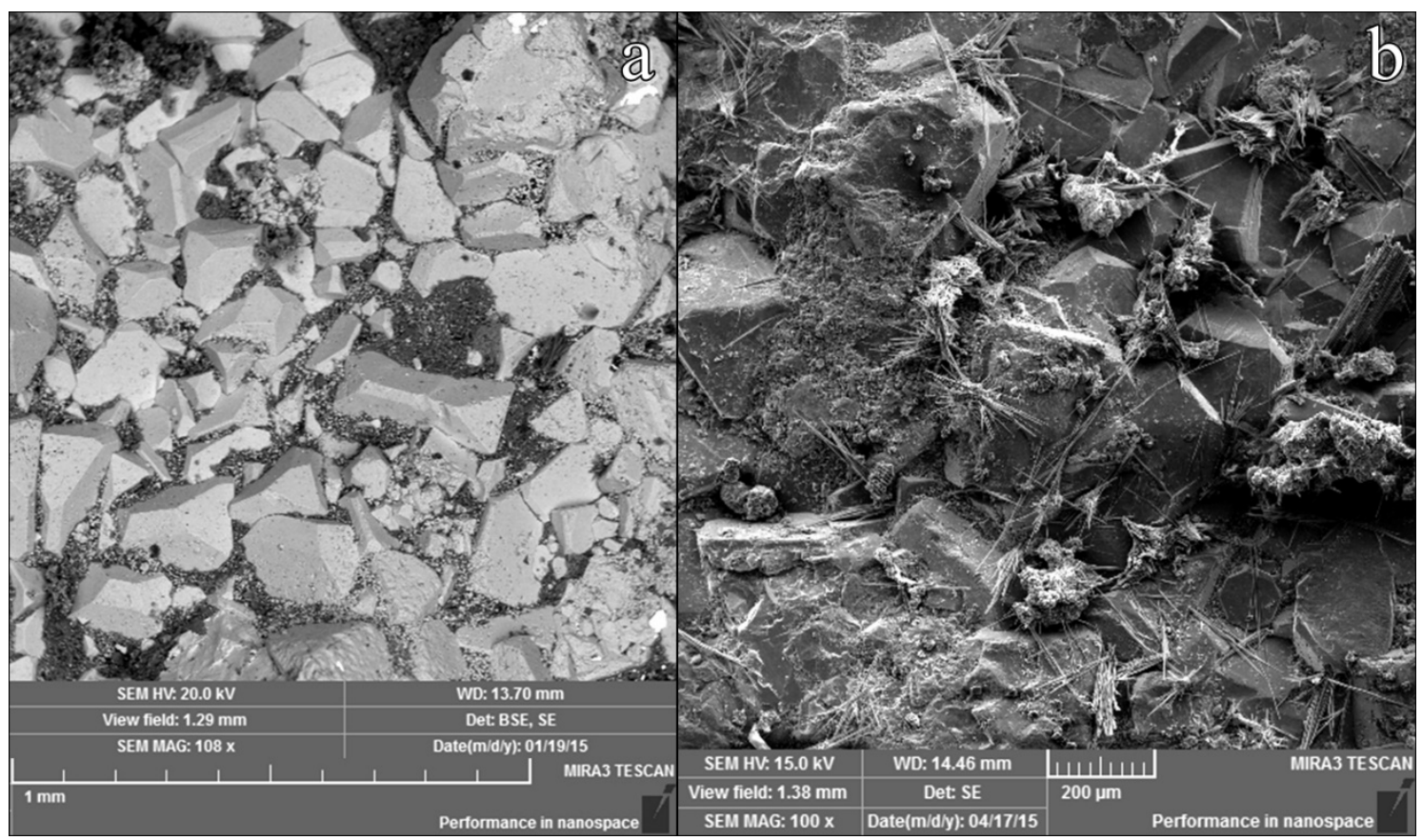

Figura 2: Análise morfológica, por SEM, da pirita com ampliação de 100x: (a) mineral à base de pirita retirada da câmara seca; e (b) a mesma amostra, exposta por 90 dias em condições ambientais controladas $\left(\mathrm{T}=(22 \pm 2){ }^{\circ} \mathrm{C}\right.$ e de UR $>50 \%)$.

Nas micrografias da Figura 3, resultado de outras ampliações da imagem da Figura 2b, foram evidenciadas distintas morfologias de cristais sobre a superfície do mineral, todas resultantes de teores diferenciados entre o enxofre e o ferro. A pirita é um sulfeto metálico suscetível ao processo de oxidação seja pela presença de $\mathrm{O}_{2}, \mathrm{H}_{2} \mathrm{O}$ ou de íons $\mathrm{Fe}^{2+}$ e $\mathrm{Fe}^{3+}$, apresentando diferentes fases intermediárias, como o $\mathrm{FeS}_{2}(\mathrm{OH})_{3}$ [37], terminando, segundo a literatura [38], na formação de tiossulfato de ferro $\left(\mathrm{FeS}_{2} \mathrm{O}_{3}\right)$.

Na Tabela 4, está disponibilizada a análise química elementar dos compostos obtidos por EDS.

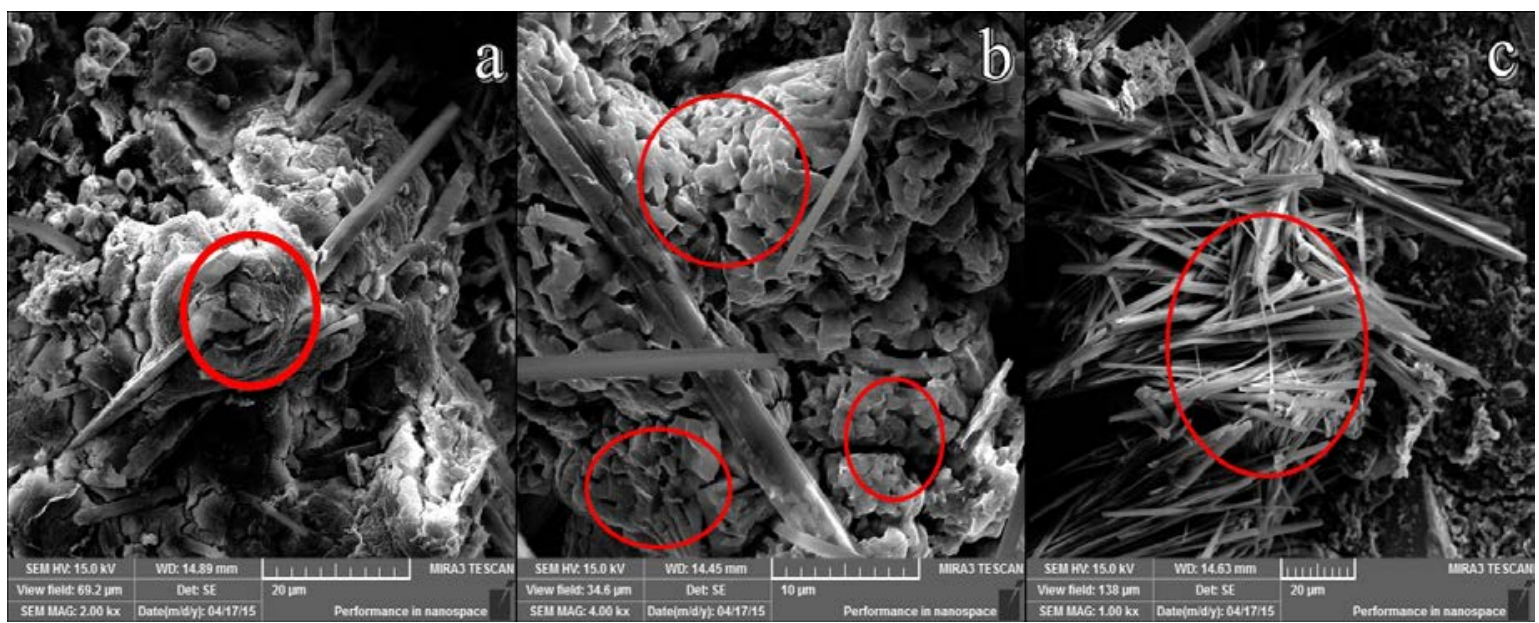

Figura 3: Imagens, por SEM, dos produtos formados pela oxidação superficial da pirita em ambiente controlado (22 \pm 2$)^{\circ} \mathrm{C}$ e de UR >50\%): (a) magnificação de 2 kx; (b) magnificação de 4 kx e; (c) magnificação de 1 kx 
PEREIRA, E; PORTELLA, K. F.; BRAGANÇA, M. O. G. P.; OCHILISKI, L. M.; OLIVEIRA, I. C. revista Matéria, v.21, n.2, pp. 342-

Tabela 4: Análise química elementar dos compostos de oxidação da pirita, obtida por EDS

\begin{tabular}{|c|c|c|c|c|c|c|}
\hline \multicolumn{6}{|c|}{ Análise química, por EDS, em \% } & \multirow{2}{*}{ Fórmula química provável (cálculo estequiométrico) } \\
\hline & $\mathbf{0}$ & $\mathbf{S}$ & $\mathrm{Fe}$ & $\mathbf{K}$ & Al & \\
\hline Figura 3a & 43,02 & 31,03 & 31,03 & - & - & $\begin{array}{l}\text { Fase intermediária de oxidação do mineral, contendo } \\
\text { em sua composição uma possível combinação química } \\
\text { dos compostos } \mathrm{Fe}_{2} \mathrm{~S}_{3}+\mathrm{Fe}_{7} \mathrm{~S}_{8} \text { [39]. }\end{array}$ \\
\hline Figura 3b & 59,85 & 17,25 & 19,81 & 1,58 & 1,52 & $\begin{array}{l}\text { Composição estequiométrica calculada, sendo equiva- } \\
\text { lente ao composto } \mathrm{Fe}_{2}\left(\mathrm{SO}_{4}\right)_{3} \text { [39]. }\end{array}$ \\
\hline Figura 3c & 59,18 & 18,66 & 16,09 & - & 6,07 & $\begin{array}{l}\text { Composição estequiométrica calculada, sendo equiva- } \\
\text { lente ao composto } \mathrm{FeS}_{2} \mathrm{O}_{3} \text { [39]. }\end{array}$ \\
\hline
\end{tabular}

Os produtos formados sobre a superfície da pirita (Figura 2b), determinados por EDS (Tabela 4) foram obtidos por cálculo estequiométrico, e os resultados corroboraram com os dados da literatura [39], mostrando diferentes estágios de oxidação. Na Figura 3a, está mostrado um produto com composição química indicativa de uma combinação de fases $\mathrm{Fe}_{2} \mathrm{~S}_{3} / \mathrm{Fe}_{7} \mathrm{~S}_{8}$ [39]; na Figura 3b, estão ilustrados cristais com relação química $\mathrm{S} / \mathrm{Fe}$, similar ao do composto $\mathrm{Fe}_{2}\left(\mathrm{SO}_{4}\right)_{3}$ [39]; e, na Figura 3c, o composto acicular $\mathrm{FeS}_{2} \mathrm{O}_{3}$ [39], o qual é a fase final de oxidação da pirita [38]. Macroscopicamente, estes produtos possuem textura pulverulenta e tonalidades variando de esbranquiçada a marrom.

A formação destes subprodutos sobre a superfície da pirita, quando o mineral é utilizado como agregado em concretos e argamassas, tende a fragilizar a interface entre a pasta de cimento e o agregado em si, reduzindo a aderência entre estas fases. Além disto, os óxidos formados podem provocar uma expansão localizada nesta região, com liberação de íons sulfato e redução do pH local, gerando condições favoráveis para a deterioração da pasta de cimento [6-10, 23-24].

\subsection{Envelhecimento natural de argamassas de cimento Portland contendo pirita}

Na Figura 4, estão apresentados os resultados de expansão relativa para barras de argamassa com e sem a presença do agregado contendo íons sulfeto. A argamassa com adição de pirita foi monitorada quanto à variação dimensional linear até que a barra retornasse ao comprimento original, relativo à primeira leitura. Para comparação, a argamassa de referência (sem pirita) foi monitorada pelo mesmo período.

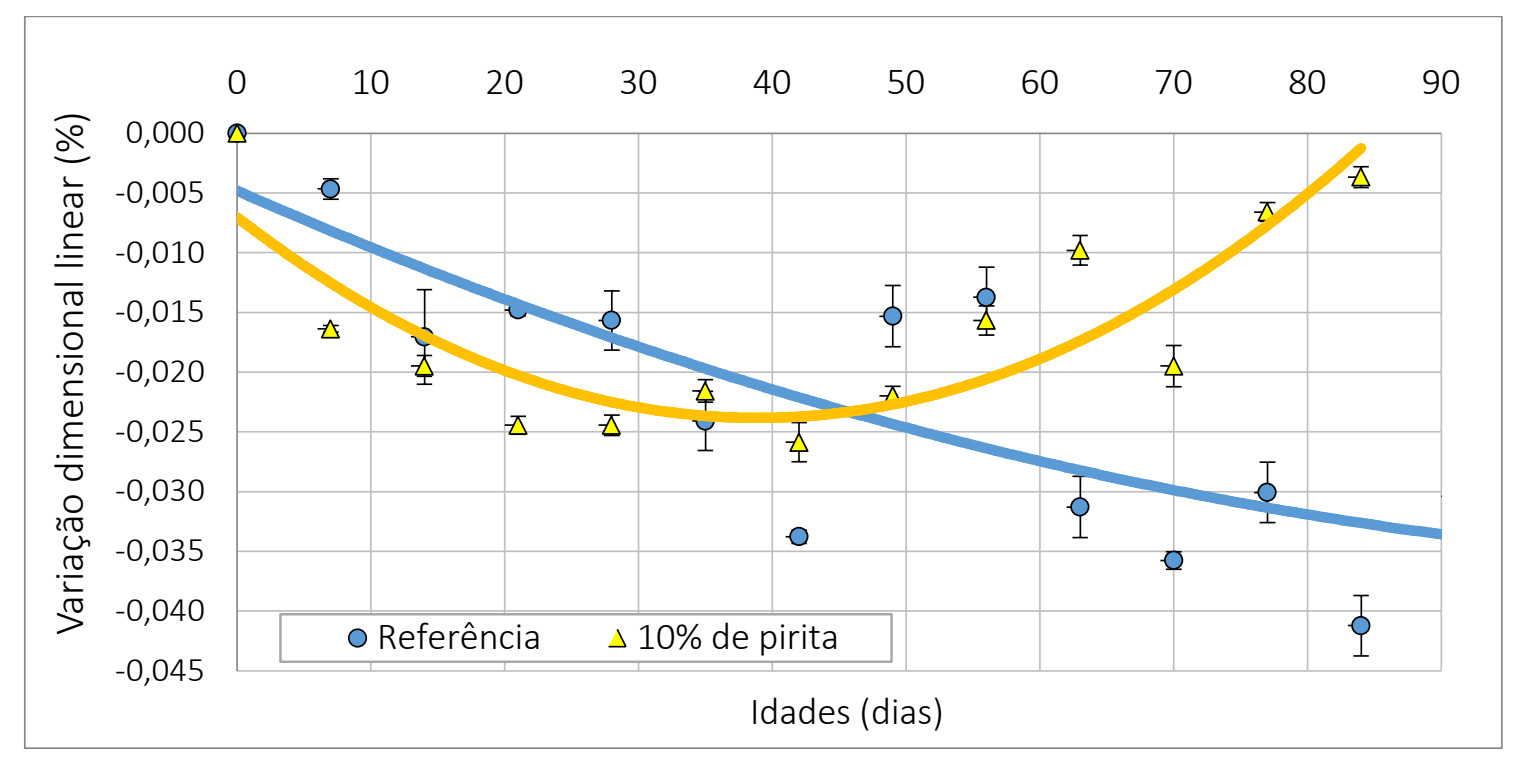

Figura 4: Gráficos da variação dimensional das barras das argamassas resultantes da variação dimensional das amostras de referência e com 10\%, em massa, de mineral contendo pirita em substituição à areia, submetidas ao envelhecimento natural.

Durante o monitoramento da variação dimensional da argamassa com adição de $10 \%$, em massa, de mineral contendo pirita, foi verificada apenas a retração das barras, sendo obtidos valores negativos de até 0,025\%. A argamassa tendeu a retornar ao comprimento inicial, relativo à primeira leitura, após 90 dias de 
envelhecimento natural. Comparativamente, isso não ocorreu de forma similar com as barras da argamassa de referência, sendo verificada somente retração até 90 dias de exposição (período avaliado). Para estas, a contração máxima verificada foi de 0,040\%.

Em estruturas de concreto ou argamassa endurecidas, podem ocorrer a retração autógena e por secagem [3, 41]. A retração autógena ocorre em função das reações químicas resultantes do processo de hidratação do cimento Portland, na qual o volume total de sólidos e líquidos no "bulk" do material diminui quando da formação dos produtos. Segundo Helene e Andrade [41], nesta fase, há auto desidratação, a qual é consequente do aumento do consumo de água livre para as reações de hidratação.

Em contrapartida, segundo os mesmos autores [41], a retração por secagem trata da perda de água da matriz cimentícia para o ambiente, sendo as variações dimensionais iniciais ocasionadas pelas perdas de água capilar, ao longo dos meses de envelhecimento natural, e de água adsorvida. Nas amostras trabalhadas, outro fator de retração foi atribuído, a auto secagem, ocasionada pela formação dos produtos etringita e gipsita, os quais contêm água de cristalização nas suas estruturas.

Apesar disto, a retração por secagem deve ser o efeito predominante nas argamassas, uma vez que estas saíram de uma condição de saturação durante os 14 dias iniciais de cura e foram expostas as condições ambientais, com umidade relativa do ar menor [36], se comparada à solução de cal. Desta forma, a água capilar ou livre tende a ser perdida e a retração ocorre. Os resultados foram consonantes com o indicado por Troxell et al. [42], que indicou que até $75 \%$ da retração por secagem ocorre após 2 semanas. Aos 42 dias, verificou-se uma inversão na tendência de retração da argamassa com 10\%, em massa, de mineral com pirita, chegando ao comprimento original aos 90 dias.

As amostras das argamassas foram analisadas por FEG SEM EDS nas idades de 42 e 90 dias, sendo os resultados apresentados nas Figuras 5 e 6, e, suas composições químicas elementares listadas na Tabela 5.

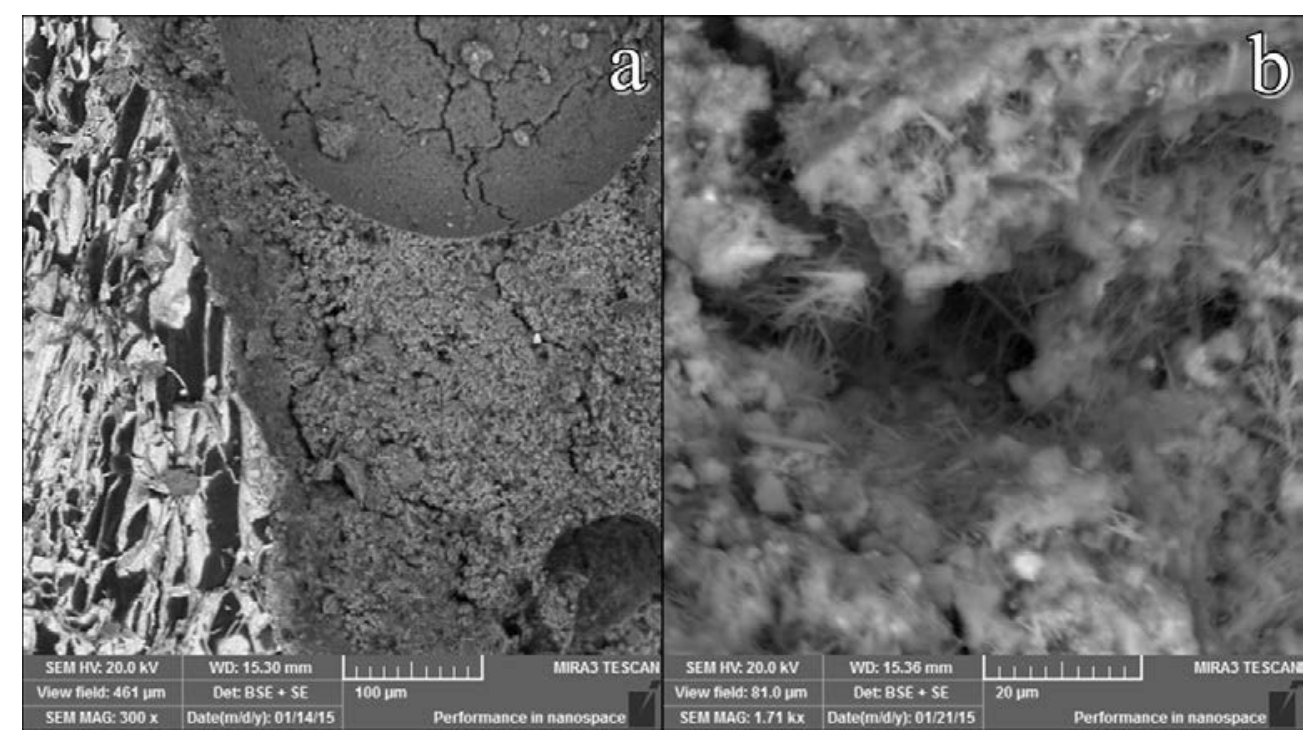

Figura 5: Microscopias das barras de argamassa aos 42 dias, com detalhamento para a (a) microfissuração da pasta e na região de interface pirita/ pasta, e (b) dos produtos formados na pasta cimentícia 


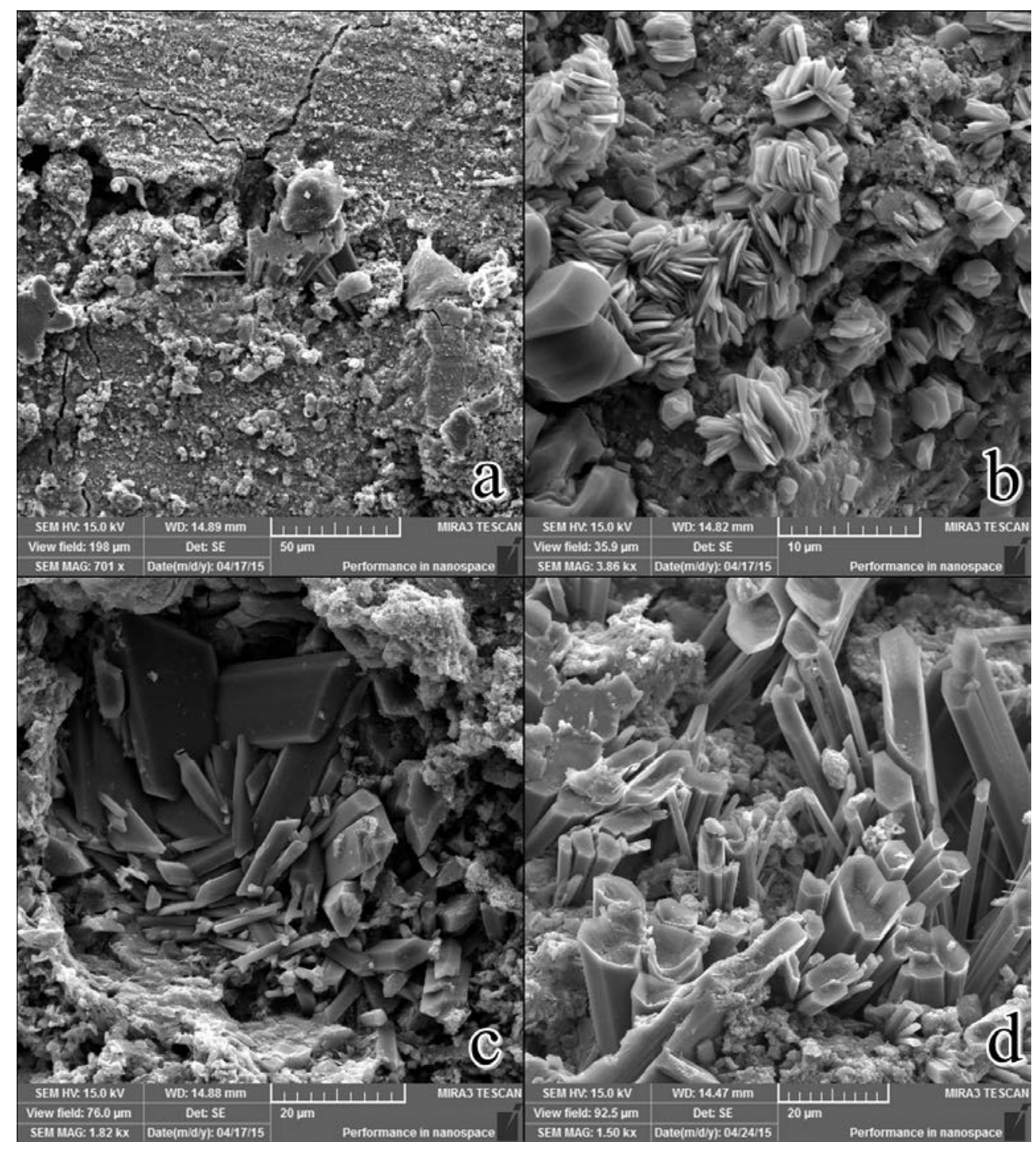

Figura 6: Microscopias das barras de argamassa aos 90 dias: (a) microfissuração da pasta; (b) óxidos de ferro formados na superfície; (c) e (d) gipsita.

Tabela 5: Análise química elementar dos compostos na pasta de cimento, obtidos por EDS

\begin{tabular}{l|l|l|l|l|l|l|l}
\hline & \multicolumn{7}{c}{ Análise química, por EDS, em \% } \\
\hline & \multicolumn{1}{|c|}{$\mathbf{0}$} & \multicolumn{1}{c|}{$\mathbf{S}$} & \multicolumn{1}{c}{ Fe } & \multicolumn{1}{c}{ Al } & \multicolumn{1}{c}{ Ca } & \multicolumn{1}{c}{ Na } & K \\
\hline Figura 5b & 58,33 & 8,45 & - & 5,05 & 28,17 & - & - \\
\hline Figura 6b & 29,81 & 27,25 & 41,71 & - & - & 0,79 & 0,44 \\
\hline Figura 6c & 42,03 & 20,24 & 0,83 & - & 36,63 & - & - \\
\hline Figura 6d & 25,93 & 30,88 & - & - & 43,19 & - & - \\
\hline
\end{tabular}

Ao analisar, por FEG SEM, a região de interface entre a pasta de cimento e a pirita na argamassa aos 42 dias (Figura 5a), verificou-se que a região é caracterizada pela presença de fissuração. Verificou-se, também, a ocorrência de cristais com morfologia de agulhas aciculares e com composição química similar à da etringita (Figura 5b e Tabela 5). A fissuração na pasta pode ter sido influenciada, provavelmente, pela formação dos produtos da oxidação da pirita, que tendem a provocar um aumento de volume na ordem de $3,05 \mathrm{~cm}^{3}$ e de $6,04 \mathrm{~cm}^{3}$ por mol de sulfeto, devido à formação do hidróxido de ferro, conforme relatado por Casanova et al. [21] e Bosch [24]. A oxidação do sulfeto forma, sobre a superfície, o hidróxido de ferro, um produto de característica pulverulenta, que pode influenciar na redução da aderência dos materiais cimentícios.

Para a argamassa, aos 90 dias, houve a formação de cristais de sulfato de ferro (relação $\mathrm{S} / \mathrm{Fe}=0,65$ ) sobre a superfície da argamassa, conforme indicado na Figura 6 b e na Tabela 5 . A presença do sulfato, em meio cimentício, tende a aumentar a ocorrência de pressões internas que podem levar à expansão devido à 
formação dos compostos descritos no caso do ataque externo, como a etringita e a gipsita [6, 22], promovendo um aumento de volume da ordem de $172 \mathrm{~cm}^{3} / \mathrm{mol}$ de sulfeto, valor superior ao observado na etapa de oxidação da pirita [21]. Na Figura 7 é apresentada imagem da barra de argamassa com 10\% de adição de pirita onde as manchas oriundas da oxidação do mineral são evidentes.

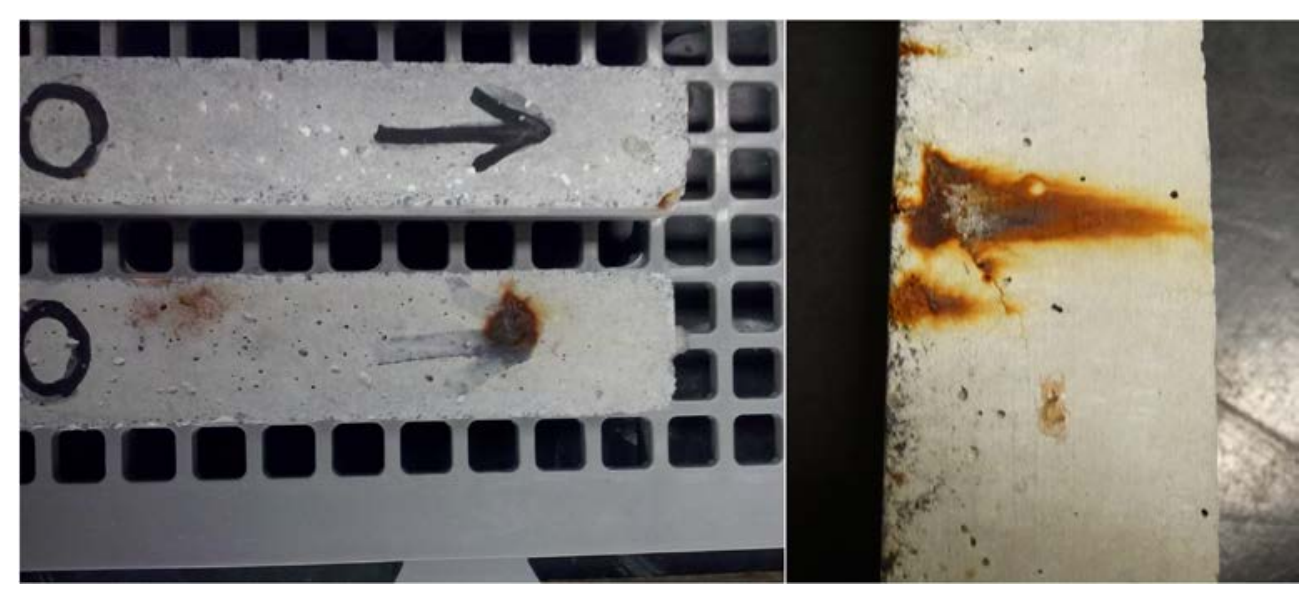

Figura 7: Barras de argamassa com adição de 10\% de pirita em substituição à areia com início de fissuração e manchas oriundas do processo de oxidação do sulfeto

Durante a oxidação da pirita, além dos óxidos liberados na superfície do mineral e lixiviados para a superfície das barras, há a disponibilização de íons sulfatos que tendem a reagir com os compostos de cimento hidratado. Esta fase é caracterizada como período de dormência, onde as reações estão ocorrendo ainda que expansões significativas não sejam verificadas.

A ocorrência de cristais de etringita foi verificada na pasta da argamassa aos 42 dias, enquanto a gipsita foi o produto predominante aos 90 dias tanto em vazios da argamassa (Figura 6c e Tabela 5) quanto na região de interface entre a pasta e o agregado (Figura 6d e Tabela 5).

A natureza dos produtos formados em materiais cimentícios preparados com os agregados contendo sulfetos depende diretamente do $\mathrm{pH}$ do sistema, o qual pode ser governado pela concentração de íons deletérios $\left(\mathrm{SO}_{4}{ }^{2-}\right.$ e $\left.\mathrm{H}^{+}\right)$liberados ao longo do processo de oxidação [42].

A formação de cristais de etringita, verificada com predominância até os 42 dias, é favorecida em ambiente cujo $\mathrm{pH}$ varia entre 10,5 e 11,5. Se os cristais de etringita estiverem disponíveis em pasta com baixa alcalinidade, eles podem ser decompostos dando origem à gipsita [43], conforme visualizado nas micrografias após 90 dias de exposição. Segundo os dados da literatura [21, 44] a gipsita é o produto predominante na superfície do material em pH inferior a 10,5. Atribuiu-se a morfologia e a composição química determinadas por SEM/EDS como consequências de uma possível redução do $\mathrm{pH}$ na região próxima à pirita em virtude da acidificação oriunda da oxidação do mineral nas condições de exposição trabalhadas.

\section{CONCLUSÕES}

A oxidação da pirita promoveu alterações morfológicas na superfície do mineral. Antes da oxidação, predominavam a ocorrência de cristais com geometrias definidas; após este processo, prevaleceram os sais à base de óxido e de sulfato de ferro.

As barras de argamassas moldadas com 10\%, em massa, do mineral contendo a pirita em substituição à areia apresentaram somente retração até os 42 dias de monitoramento em envelhecimento natural, atingindo valores de até $-0,025 \%$. No entanto, macroscopicamente, verifica-se nas barras de argamassa a presença de manchas marrom típica do processo de oxidação da pirita.

A análise morfológica, após esta idade, indicou que a oxidação do mineral afetou a microestrutura da interface entre a pasta de cimento e o agregado, levando à redução da aderência na região e à nucleação e crescimento de cristais de etringita. A formação destes cristais provocou a ocorrência de microfissuração na matriz cimentícia.

Aos 90 dias houve a predominância de cristais de gipsita na microestrutura da pasta e a formação de sulfato e de óxidos de ferro com geometrias bem definidas. Este comportamento deveu-se à oxidação parcial e total da pirita com consequente acidificação da região, o que provocou mudanças de pH na argamassa. Macroscopicamente, além das manchas superficiais na argamassa oriundas das reações de oxidação é possível 
verificar a ocorrência de fissuras nas regiões próximas ao sulfeto, indicando que apesar de não haver expansão das barras de argamassa o ataque interno por sulfetos está ocorrendo, caracterizando-se assim o período de dormência do ataque.

\section{AGRADECIMENTOS}

Os autores agradecem à infraestrutura e ao apoio em recursos humanos e financiamento à COPEL GeT projeto P\&D 6491-0301/2013, à ANEEL, ao CNPq Lei 8010/90, principalmente a LI 14/344508-1 DI 14/2190047-7, aо CNPq PIBITI e Bolsa Produtividade em pesquisa 305718/2011-8, aos Institutos LACTEC, a Universidade Federal do Paraná - PIPE/UFPR e ao C-LAMU da Universidade Estadual de Ponta Grossa UEPG.

\section{BIBLIOGRAFIA}

[1] HASPARYK, N. P., NASCIMENTO, J. F. F., ANDRADE, M. A. S., et al, "Estudos de laboratório com concretos contendo agregados obtidos a partir de rocha com sulfetos”, In: Reunión Técnica de la AATH Seminario sobre Hormigones Especiales, 15a , Argentina, 2003.

[2] BELZILE, N., CHEN, Y. W., CAI, M. F. et al, “A review on pyrrhotite oxidation”, Journal of Geochemical Exploration, v. 84, pp. 65-76, 2004.

[3] HASPARYK, N. P., GOMIDES, M. J., ANDRADE, M. A. S., et al., "Estudo em laboratório de concretos contendo agregados com sulfetos”, In: Congresso Brasileiro do Concreto, 47², 2005, Recife.

[4] KLEIN, C., DUTROW, B., “Manual de ciência dos minerais”, Porto Alegre, Bookman, 2012.

[5] FORADADA, J. S., Envejecimiento de presas por reacciones expansivas en hormigón, Minor thesis Universitat Politècnica de Catalunya, Espanha, 2005.

[6] ARAUJO, G. S., Comportamiento de presas de gravedad de hormigón con problemas de ataque interno por sulfatos, Tesis Doctoral, Universitat Politècnica de Catalunya, Espanha, 2008.

[7] OLIVEIRA, I., “Reacción sulfáticas interna en presas de hormigón: cinética Del comportamento”, 2011. Tesis Doctoral - Departament d’Enginyeria de la Construcció, Universitat Politècnica de Catalunya, Espanha.

[8] MOURA, A. C., Análisis numérico de presas de hormigón bajo acciones expansivas, Tesis doctoral Departament d’Enginyeria de la Construcció, Universitat Politècnica de Catalunya, Espanha, 2012.

[9] BRUNETAUD, X., KHELIFA, M. R., AL-MUKHTAR, M., "Size effect of concrete samples on the kinetics of external sulfate attack”, Cement and Concrete Composites, v. 34, pp. 370-376, 2012.

[10] DUCHESNE, J., FOURNIER, B., "Deterioration of Concrete by the Oxidation of Sulphide Minerals in the Aggregate”, Journal of Civil Engineering and Architecture, v. 7, n. 8, pp. 922-931, 2013.

[11] FONSECA, D. S., BASIC, I. R., "Enxofre”, In:

https://sistemas.dnpm.gov.br/publicacao/mostra_imagem.asp?IDBancoArquivoArquivo=4005, 577-590, acessado em maio de 2015.

[12] ASSOCIAÇÃO BRASILEIRA DE NORMAS TÉCNICAS. NBR 13583: Cimento Portland - Determinação da variação dimensional de barras de argamassa de cimento Portland expostas à solução de sulfato de sódio. Rio de Janeiro, 2014.

[13] ALMEIDA, C. M. V. B., “Estudo eletroquímico das interações entre sulfetos de ferro”, Tese de D.Sc., , Instituto de Química, da Universidade de São Paulo, São Paulo, Brasil, 1999.

[14] CALDEIRA, C. L., Investigation of pyrite oxidation in alkaline solutions by thermodynamic modeling and analyses by X-ray diffraction, infrared and Raman spectroscopy, Tese de D.Sc., Curso de PósGraduação em Engenharia Metalúrgica e de Minas, da Universidade Federal de Minas Gerais, Belo Horizonte, 2005.

[15] ROISENBERG, C., FORMOSO, M. L. L., DANI, N., et al., "Caracterização e evolução geoquímica das águas subterrâneas da mina de Candiota (RS), Brasil”, Revista Brasileira de Geociências, v. 38, n.4, São Paulo, 2008.

[16] GOMIDES, M. J., "Investigação de agregados contendo sulfetos e seus efeitos sobre a durabilidade do concreto”, Tese de D.Sc., Programa de pós-graduação em engenharia civil, da Universidade Federal do Rio Grande do Sul, Porto Alegre, 2009. 
[17] CHINCHÓN-PAYÁ, S., AGUADO, A. S., CHINCHÓN, “A comparative investigation of the degradation of pyrite and pyrrhotite under simulated laboratory conditions”, Engineering Geology, v. 127, pp. 75-80, 2012.

[18] MA, Y., LIN, C., "Microbial Oxidation of Fe2+ and Pyrite Exposed to Flux of Micromolar H2O2 in Acidic Media”, Scientific Reports, 3, 1979, 2013.

[19] GOMIDES, M. J., HASPARYK, N. P., ANDRADE, M. A. S., et al., "Investigação de agregados contendo sulfetos para utilização em estruturas de concreto”, In: XXVI Seminários nacional de grandes barragens, Goiânia, 2005.

[20] NEVILLE, A. M., Propriedades do concreto, Trad. Salvador E. Giammusso, São Paulo, Pini, 1997.

[21] CASANOVA, I., AGULLÓ, L., AGUADO, A., "Aggregate expansivity due to sulfide oxidation - I. Reactions system and rate model”, Cement and Concrete Research, v. 26, n. 7, pp. 993-998, 1996.

[22] MEHTA, P. K., MONTEIRO, P. J. M., “Concreto: estruturas, propriedades e materiais”, São Paulo, Pini, 1ed, 2008.

[23] WERITZ, F., TAFFE, A., SCHAURICH, D., et al., "Detailed depth profiles of sulfate ingress into concrete measured with laser induced breakdown spectroscopy", Construction and building materials, v. 23, pp. 275 - 283, 2009.

[24] BOSCH, F. P., "Estudio del diagnóstico y del tratamiento de presas de hormigón con expansiones”, Projecte o tesina d'especialitat, Escola Tècnica superior d'Enginyers de Camins, Canls i Ports de Barcelona, Universitat Politécnica de Catalunya, Espanha, 2009.

[25] BICZOK, I., ASENSI, E. J. D., “Corrosion y proteccion del hormigon”, URMO, S. A. de Ediciones, Bilbao, Espanha, 1981.

[26] http://www.cimentoitambe.com.br/relatorios-de-ensaio/?pro=368\&chave=2014-7, acessada em setembro 2014.

[27] ASSOCIAÇÃO BRASILEIRA DE NORMAS TÉCNICAS. NBR NM 52: Agregado miúdo - Determinação da massa específica e massa específica aparente. Rio de Janeiro, 2009.

[28] ASSOCIAÇÃO BRASILEIRA DE NORMAS TÉCNICAS. NBR NM 30: Agregado miúdo - Determinação da absorção de água. Rio de Janeiro, 2001.

[29] ASSOCIAÇÃO BRASILEIRA DE NORMAS TÉCNICAS. NBR NM 45: Agregados - Determinação da massa unitária e do volume de vazios. Rio de Janeiro, 2006.

[30] ASSOCIAÇÃO BRASILEIRA DE NORMAS TÉCNICAS. NBR NM 46: Agregados - Determinação do material fino que passa através da peneira 75 um, por lavagem. Rio de Janeiro, 2003.

[31] LIMA, A. L. C., "Influência da presença de sulfetos na implantação da UHE Irapé - Vale do Jequitinhonha - Minas Gerais”, Dissertação de M.Sc., Núcleo de Geotecnia da Escola de Minas, Universidade Federal de Ouro Preto, Ouro Preto, Brasil, 2009.

[32] HAGERMAN, T., “Chemical Reactions Involving Aggregate: Discussion”, In: Chemistry of Cement: Proceedings of the Fourth International Symposium, v. II, Washington, 1960.

[33] WAKIZAKA, K., ICHIKAWA, Y., NAKAMURA, S., "Deterioration of concrete due to specific minerals”, Environment and Economy, v. 2, pp. 331-338, 2001.

[34] FAQUINELI, C. R., "Estudo da reatividade de agregados contendo sulfetos em concretos e argamassas", Dissertação de M.Sc., Programa de Pós-Graduação em Engenharia de Estruturas, da Escola de Engenharia da Universidade Federal de Minas Gerais, Belo Horizonte, Brasil, 2010.

[35] BRAGANÇA, M. D. G. P., PORTELLA, K. F., BONATO. M, M., et al., "Estudo de Manifestação Patológica por Sulfato em Argamassa Preparada com Agregado Artificial Reativo”, In: Congresso Brasileiro do Concreto, 55, Gramado, 2013.

[36] INMET. Disponível em: http://www.inmet.gov.br/sim/gera_graficos.php, acessada em maio 2015.

[37] GUIMARÃES, L., "Estudo da especiação química do Fe3+ e Fe2+ em meio aquoso a partir de cálculo DFT: implicações para o mecanismo de oxidação da pirita”, Dissertação de M.Sc., Universidade Federal de Minas Gerais, Belo Horizonte, 2007.

[38] GUEVARA, L. R. Z., "Predição do potencial de drenagem ácida de minas utilizando o método cinético da coluna de lixiviação”, Dissertação de M.Sc, Pontifícia Universidade Católica do Rio de Janeiro, Rio de Janeiro, 2007. 
[39] OLIVEIRA, I., CAVALARO, S. H. P., AGUADO, A., "Evolution of pyrrhotite oxidation in aggregates for concrete”, Materiales de Construcción (Madrid, Spain), v. 64, n. 316, pp. 1-9, 2014.

[41] HELENE, P., ANDRADE, T. W. C. O., Materiais de Construção Civil e Princípios de Ciência e Engenharia de Materiais, São Paulo, IBRACON, 2007.

[42] TROXELL, G.E., RAPHAEL, J.E. AND DAVIS, R.W., "Long-time creep and shrinkage tests of plain and reinforced concrete”, Proc. ASTM 58, pp.1101-1120, 1958.

[43] AYORA, C., CHINCHÓN, S., AGUADO, A., et al., "Weathering of iron sulfides and concrete alteration: thermodynamic model and observation in dams from central Pyrenees, Spain”, Cement and Concrete Research, v. 28, n. 9, pp. 1223-1235, 1998.

[44] SANTHANAM, M., COHEN, M. D., OLEK, J. "Sulfate attack research - whither now?”, Cement and Concrete Research, v. 31. pp. 845 - 851, 2001. 